\title{
Effects of a 5-Week Linear Periodization Program on Maximal Strength and Speed on Elite Squash Players
}

\author{
Jorg Teichmann', Allan Pete Soyzaㄹ, Ali Habib Harb², Kieran Stefan Allan', Rachel Tan Ee ${ }^{1}$ \\ ${ }^{1}$ Rehamed Therapy, Shah Alam, Malaysia \\ ${ }^{2}$ Ranald Joseph National Sport Institute of Singapore, Singapore, Singapore \\ Email: jorg@rehamedtherapy.com.my
}

How to cite this paper: Teichmann, J., Soyza, A. P., Harb, A. H., Allan, K. S., \& Ee, R. T. (2021). Effects of a 5-Week Linear Periodization Program on Maximal Strength and Speed on Elite Squash Players. Advances in Physical Education, 11, 369-386. https://doi.org/10.4236/ape.2021.114031

Received: July 18, 2021

Accepted: September 26, 2021

Published: September 29, 2021

Copyright $\odot 2021$ by author(s) and Scientific Research Publishing Inc. This work is licensed under the Creative Commons Attribution International License (CC BY 4.0).

http://creativecommons.org/licenses/by/4.0/

\begin{abstract}
Context: Participation in squash requires high efficiency in agility, speed, reaction time and repetitive muscular endurance, aerobic and anaerobic capacities. Standard traditional periodization to reach optimal physical fitness for squash is limited. Therefore, this study aims to find a more alternative approach in training design. Objective: The purpose of this article aims to highlight the benefits of a 5-week linear strength and conditioning program to help improve strength and speed in elite squash players. Design: Case study. Setting: Field research. Participants: 3 male and 2 female Singapore elite squash players in preparation for the 2019 South East Asian Games. Intervention(s): 5-week linear periodization program was a sport specific injury prevention training with a total of 12 special strength sessions, 4 hypertrophy sessions, and 3 speed and plyometric sessions. Main Outcome Measure(s): 1) Maximal strength tests, the athletes were guided into performing a 1-repetition max (RM) half squat test, 1RM single leg adductor test, 2) muscular endurance tests,1-minute push up test, 1-minute sit up test, and 3) maximal speed tests, a 20-meter Sprint test. Tests were conducted before training program and after 5-week linear periodization program. Results: Significant improvement for 1-RM half squat test, 1RM single leg adductor test, 1-minute push up test, 1-minute sit up test and 20 -meter sprint test were noted after 5 weeks of linear periodization program. There were significantly higher rates of improvement in strength compared to speed. Conclusions: 5-week linear periodization training program displayed significant improvement in strength, muscular endurance and speed suggesting that a linear strength and conditioning program can be highly beneficial for squash players.
\end{abstract}




\section{Keywords}

Periodization, Linear Periodization, Squash, Strength and Conditioning, Strength, Power, Speed, Injury Prevention

\section{Introduction}

Squash is an extremely challenging competitive sport that requires an enormous level of physical fitness to compete on an elite level. Repetitive short explosive movements with split second responses and decisions to compensate for high ball travel speeds recording up to $281.6 \mathrm{~km} / \mathrm{h}$ require high efficiency in agility, speed and reaction time pertaining to skill related fitness components. In squash, the ability to perform rapid acceleration and deceleration over short distances is essential for success where power plays an important role. Power is defined as the rate at which work is done or energy is transferred to perform a task (van der Kruk, van der Helm, Veeger, \& Schwab, 2018) or the ability to exert maximal force in a short period of time. Research has shown that maximal strength is a major factor influencing performance and stronger athletes are found at higher levels of competition in a variety of sports compared with weaker athletes (Haff \& Nimphius, 2012).

Participation in squash requires high competency in a variety of parameters such as muscular endurance, aerobic and anaerobic capacities, strength, power, speed, and agility (Bennie \& Hrysomallis, 2005). Heart rates are often at an average of $190 \mathrm{bpm}$ spiking above thresholds of $80 \%-85 \%$ of the players predicted maximum heart rate (Docherty, 1982) due to the intensive nature of the sport that can come up to rallies lasting $45 \mathrm{~s}-60 \mathrm{~s}$ with very short breaks in between. Players may cover up to 5000 meters in a single 5 set match of squash lasting anywhere between 60 to 90 minutes requiring excellent cardiovascular endurance.

Literature concerning methods of training interventions to reach an elite level of physical fitness for squash is minimal. Typically, in a linear periodization, different training phases last approximately 4 - 6 weeks generally showing a steady progression with increasing intensities and decreasing volumes (Fleck, 2011). In this case study, the subjects consisted of high-performance elite athletes with different neuromuscular adaptations requiring variation from typical training methods. To ensure progress is made over a long period of time, some type of periodization or program variation is required (Kraemer, Duncan, \& Volek, 1998). The limitations of standard traditional periodization raise hurdles to further progress (Issurin, 2010). In recent times, more alternative approaches in training design have appeared with little scientific deliberation.

In more highly trained athletes, strength gains are not achieved with low volume single-set training programs (Willoughby, 1993) therefore our program sees an increase in both volume and intensity over time. The cross-sectional area and percentage of fibre types within a muscle will vary significantly among elite athletes. The possibility exists to modify the characteristics of skeletal muscle if 
the stimulus presented is sufficient (Kraemer, Duncan, \& Volek, 1998). This article aims to highlight the benefits of a 5-week linear strength and conditioning program to help improve strength and speed in elite squash players.

\section{Methodology}

3 male and 2 female elite squash players were subjected to a 5-week linear periodization program in preparation for the 2019 South East Asian Games. During the training program the athletes were subjected to maximal strength tests, muscular endurance tests and maximal speed tests. The athletes were guided into performing a 1-repetition max (RM) half squat test, 1-minute push up test, 1RM single leg adductor test, 1-minute sit up test and a 20-meter Sprint test.

Before beginning any tests, the athletes were given 30 minutes to warm up. The warm-up phase included light cardio on a treadmill or stationary bike followed by dynamic stretches and mobility to ensure the athletes were adequately prepared.

Table 1 breaks down how each athlete was given 3 - 5 sets in order to determine the athlete's 1RM. The athlete was then given $2-3$ attempts in order to determine the athlete's $1 \mathrm{RM}$. Table 2 describes the fitness components targeted and description of some examples of exercises given based off the list in Table 1.

- Half Squat Test (HST)

Athletes were instructed to stand, hip width apart, behind the barbell. When cued, the athletes picked up the barbell, standing tall in the squat rack. The weight of the barbell started at $80 \%$ of the $1 \mathrm{RM}$ of each athlete from a previous test conducted the year before. The athletes were then instructed to do a half squat. The weights were gradually increased every set until 1RM was achieved.

Table 1. List of SSIPT exercises, prescribed sets and reps, and rest time.

\begin{tabular}{cccc}
\hline EXERCISE & SETS & REPS/SEC & REST \\
\hline Resisted Static Squats & 5 & $20 \mathrm{Reps}$ & $10 \mathrm{Sec}$ \\
SLER Banded Rows & 6 & $15 \mathrm{Reps}$ & $0 \mathrm{Sec}$ \\
SL Banded Jumps & 5 & $12 \mathrm{Reps}$ & $0 \mathrm{Sec}$ \\
Resisted Explosive Reverse Lunges & 8 & $20 \mathrm{Sec}$ & $10 \mathrm{Sec}$ \\
5 Hurdle Jump & 2 & $8 \mathrm{Reps}$ & $3 \mathrm{Min}$ \\
Bosu Banded Stars & 8 & $10 \mathrm{Reps}$ & $10-20 \mathrm{Sec}$ \\
RLE Barbell Lunge & 8 & $10 \mathrm{Reps}$ & $0 \mathrm{Sec}$ \\
Static Shoulder Abd. With Banded Leg Add. & 6 & $10 \mathrm{Reps}$ & $0 \mathrm{Sec}$ \\
Ant. Banded Static Squat With Medball Press & 10 & $10 \mathrm{Rep}$ & $0 \mathrm{Sec}$ \\
Posterior Banded Static Squat with Medball Press & 10 & $10 \mathrm{Rep}$ & $0 \mathrm{Sec}$ \\
Bosu Banded Superman & 6 & $10 \mathrm{Reps}$ & $10-20 \mathrm{Sec}$ \\
Sit-Ups & 8 & $20 \mathrm{Reps}$ & $10 \mathrm{Sec}$ \\
\hline Elevated Single Leg Swiss Ball Bridge & 6 & $10 \mathrm{Reps}$ & $0 \mathrm{Sec}$ \\
\hline
\end{tabular}


- Single Leg Adductor test:

The athletes were instructed to lie on the side which is being tested. The untested leg is then flexed to $90^{\circ}$ at the hip and knee joint while the ankle is maintained in a neutral position. The tested leg is kept straight with $0^{\circ}$ hip and knee flexion. Ankle weights were placed around the malleolus of the tested leg. The athlete was then instructed to lift the leg vertically upwards (adduction in side-lying) by trying to overcome the resistance of the ankle weights while maintaining the ankle in a neutral position. The weights were gradually increased. The process was performed on both legs.

- 1 Minute Push-up Test (PUT)

The test began with the athlete in a push-up position, hands placed shoulder width apart with elbows in full extension. The athlete was asked to maintain a neutral spine throughout the movement. A 4-inch block was positioned directly below the athletes' chests to standardize push up depth every time. 1 repetition was counted when the athlete performs a push up and the chest comes into contact with the block and then returning to the starting position. Each athlete was given 1 minute to perform a maximum number of repetitions.

- 1 Minute Sit-up Test (SUT)

The athlete began in a supine position with both knees flexed to $90^{\circ}$, feet on the ground. The athlete was instructed to sit up and reach to a marker that has been measured to $20 \mathrm{~cm}$ from the fingertips and directly above the knees. A repetition is counted when the athlete performs a sit up, makes contact with the markers and then the head returns to the starting position on the floor.

- $5 \mathrm{~m}, 10 \mathrm{~m}, 15 \mathrm{~m}$ and $20 \mathrm{~m}$ Sprint Test

For this test, a wireless timing gate system (Swift Performance Timing Gate) was set up on a 50 meter length track. The timing gates where set up at $5 \mathrm{~m}, 10$, $15 \mathrm{~m}$ and $20 \mathrm{~m}$ intervals. A finish line was placed at the 25 -meter mark and all athletes were advised to not slow down until they reach the $25 \mathrm{~m}$ mark to ensure the results are not compromised by early deceleration.

Each athlete begins less than 1 foot away from the first-time gate and were instructed to start sprinting when ready. Each athlete was given a total of 5 attempts with 3 minutes break between each sprint. The best possible time for $5 \mathrm{~m}$, $10 \mathrm{~m}, 15 \mathrm{~m}$ and $20 \mathrm{~m}$ were recorded for each athlete and displayed in Table A3.

\section{Training Program Outline}

A 5-week training program was designed to help improve overall strength, acceleration, and explosive power in squash players. During the 5-week training program, the athletes underwent a total of 12 special strength sessions, 4 hypertrophy sessions, and 3 speed and plyometric sessions.

- Sport Specific Injury Prevention Training (SSIPT)

SSIPT can be defined as the concept of applying sport specific exercises to help develop specific strength and reduce injury to carry out a specific task or sport. 
Data captured from a case study following the UCS Swedish Open 2018 utilizing the Motrack system "captured players covering distances upwards of 1 kilometre per single game, with matches played in a best-of-five-game format, comprising hundreds of changes in direction and multiple complex movements, with player heart rates regularly registering upwards of $190 \mathrm{bpm}$-at times almost hitting 200 bpm" ("Data Highlights Squash's True Physical Demands|Professional Squash Association”, 2021).

The SSIPT exercises chosen are specifically designed to mimic various aspects of squash which includes lunges, backward movements, rapid acceleration \& deceleration, explosive power, functional core stability and aerobic endurance. Each session included 13 various exercises targeting joint stability, movement patterns, core stability and basic explosive power.

- Week 1 \& 2 Summary

The athletes underwent a total of 6 Special Strength Training (SST) sessions for the first 2 weeks which was intended on improving sport specific movements. Each session included 12 various exercises targeting joint stability, movement patterns, core stability and basic explosive power. The athletes also underwent 2 strength sessions only utilizing a medicine ball. The medicine ball sessions included a total of 150 throws divided into various movements (wall slams, overhead throws, floor slams, etc.).

- Weeks 3, 4 \& 5 Summary

During the $3^{\text {rd }}, 4^{\text {th }}$ and $5^{\text {th }}$ week of the training program the athletes underwent a total of 6 SST sessions, 4 muscular hypertrophy sessions including the session from the $2^{\text {nd }}$ week. The players also underwent a total of 3 speed and plyometric sessions.

The muscular hypertrophy sessions included 8 to 9 exercises in each session. The first 4 exercises were always compound lifts including Squats, Bench Press, Dead Lifts and Overhead Press. Minor adaptions were conducted from session to session by adjusting either the number of sets or intensity. Table 3 shows the reps, sets, and intensity used during the hypertrophy sessions.

The speed and plyometric sessions were divided into 2 parts. The initial part was 3 sets of 10 sprints for 15 meters. After each 15 -meter sprint the player would jog back to the starting point and immediately begin the next sprint until completing 10 consecutive sprints. Each player was given 3 minutes rest between each set.

The plyometric phase included 3 sets of basic hurdle jumps. 6 hurdles were set up 5 feet apart while the height of each hurdle was adjusted according to the height of each players' greater trochanter. 6 repetitions of 6 jumps constitute a single set. Each player was given 6-minute rests in between each set and no rest in between repetitions.

- Retest at 5 Weeks

After completing the 5-week training program, the athletes were retested for their strength and speed to assess their levels of improvement throughout the training program. All the participating athletes demonstrated positive increases 
Table 2. Examples of exercises and descriptions based on targeted fitness component.

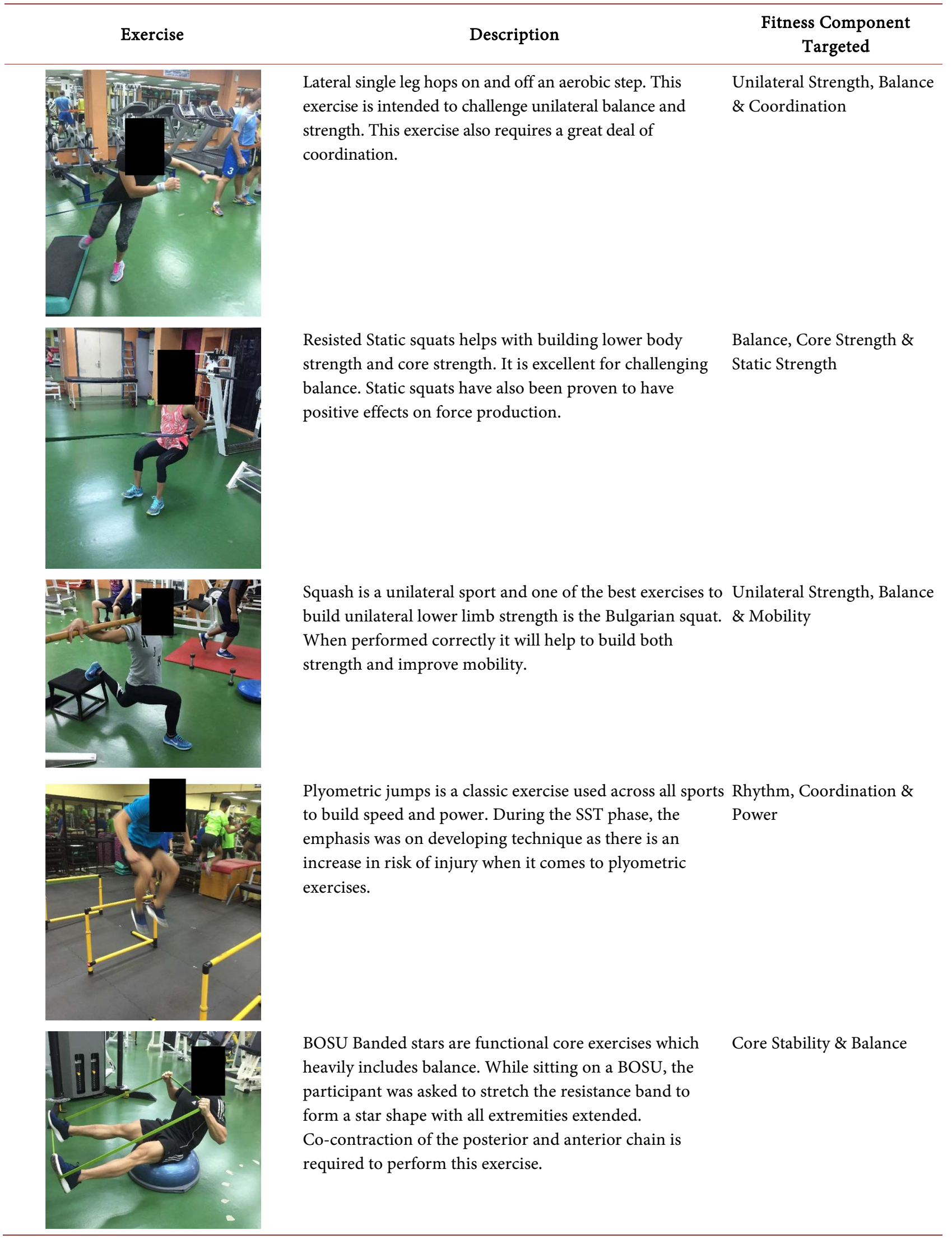


Table 3. Guideline set for sets and intensity.

\begin{tabular}{cccc}
\hline Hypertrophy & Sets & Reps & Intensity \\
\hline Session 1 & 3 & 8 & $65 \%$ \\
Session 2 & 4 & 8 & $65 \%$ \\
Session 3 & 5 & 8 & $65 \%$ \\
Session 4 & 5 & 8 & $75 \%$ \\
\hline
\end{tabular}

in both strength and speed following the 5-week training program. There were significantly higher rates of improvement in strength compared to speed. Pre-post comparison results can be seen in Figure 1, Figure 2, Figure 3, Figure 4, and Figure 5.

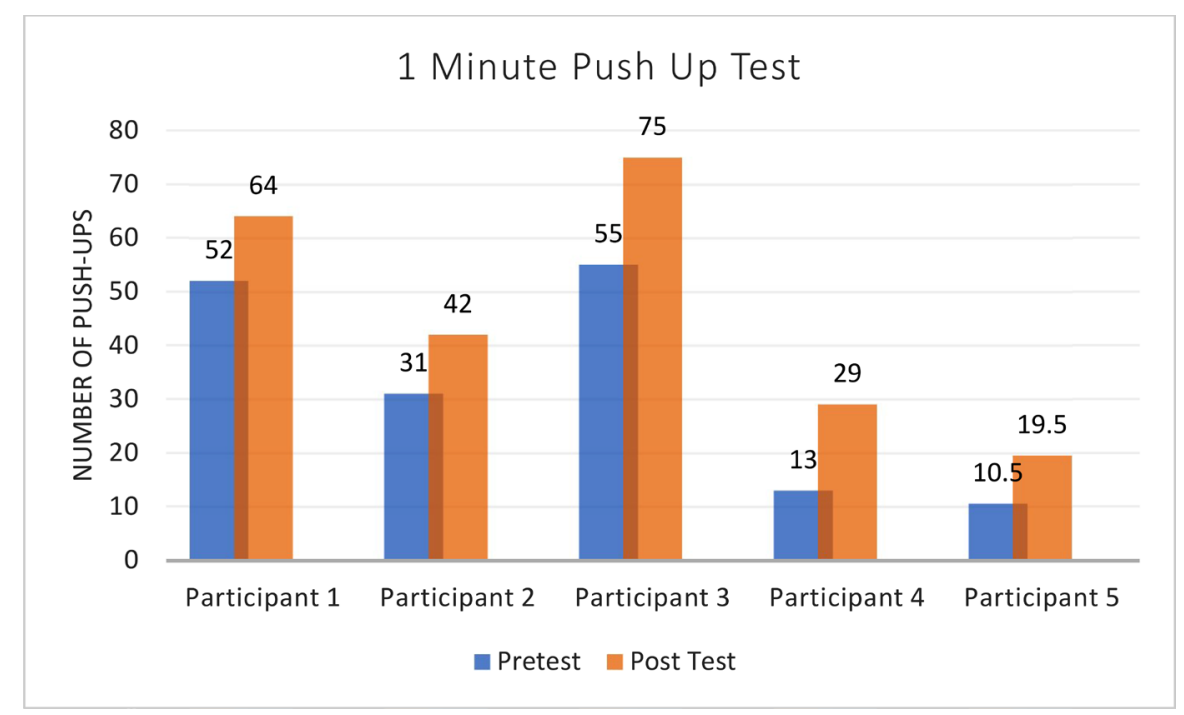

Figure 1. Pre- and post-test results comparison of the 1-minute push up test.

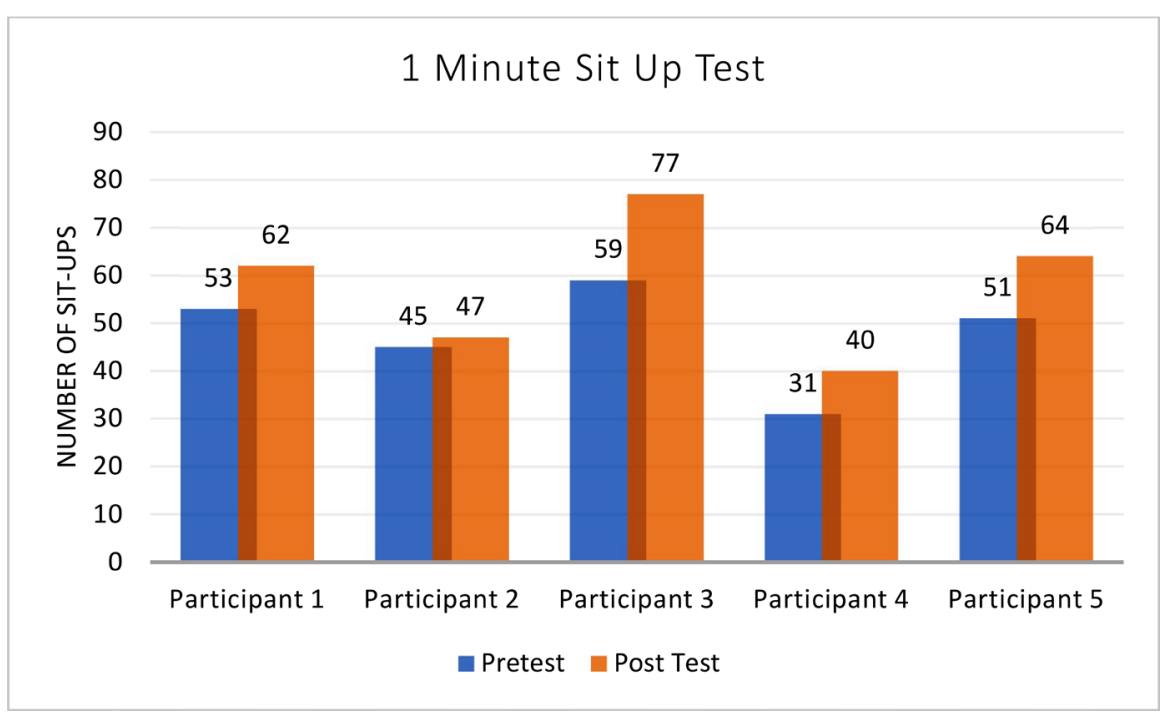

Figure 2. Pre- and post-test results comparison of the 1-minute sit up test. 


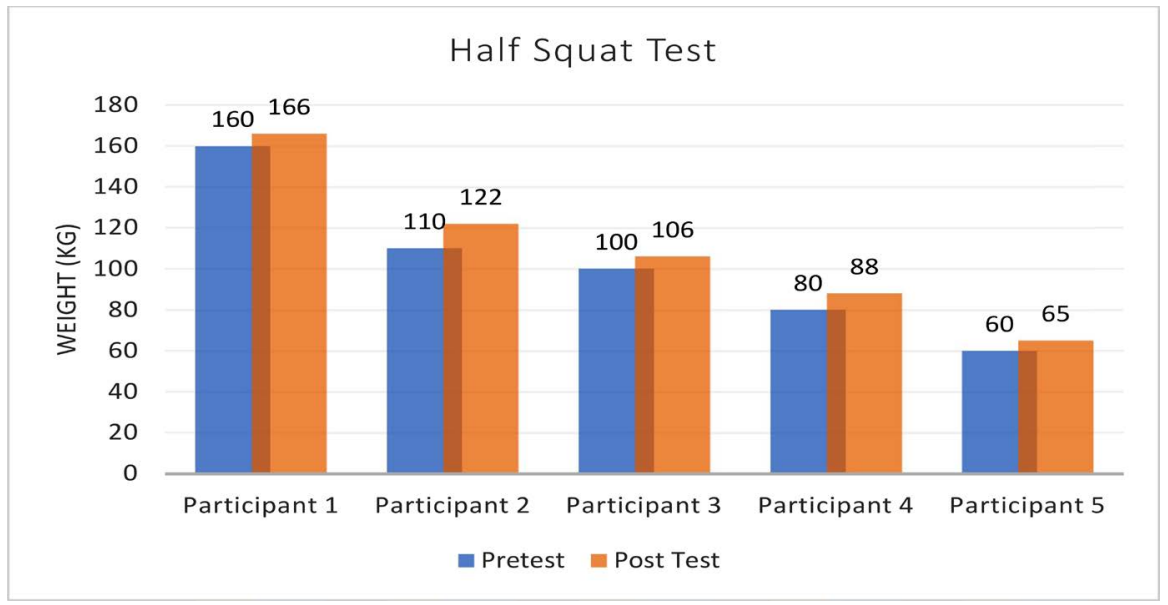

Figure 3. Pre- and post-test results comparison of the half squat test.

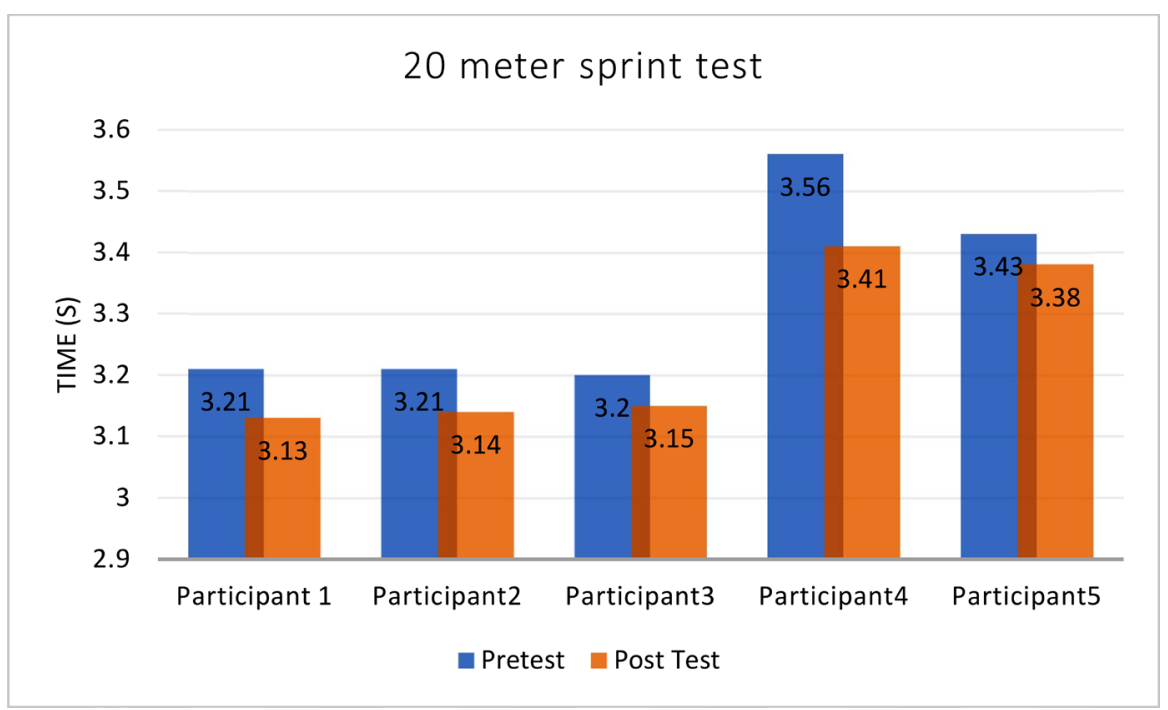

Figure 4. Pre- and post-test results comparison of the 20-meter sprint test.

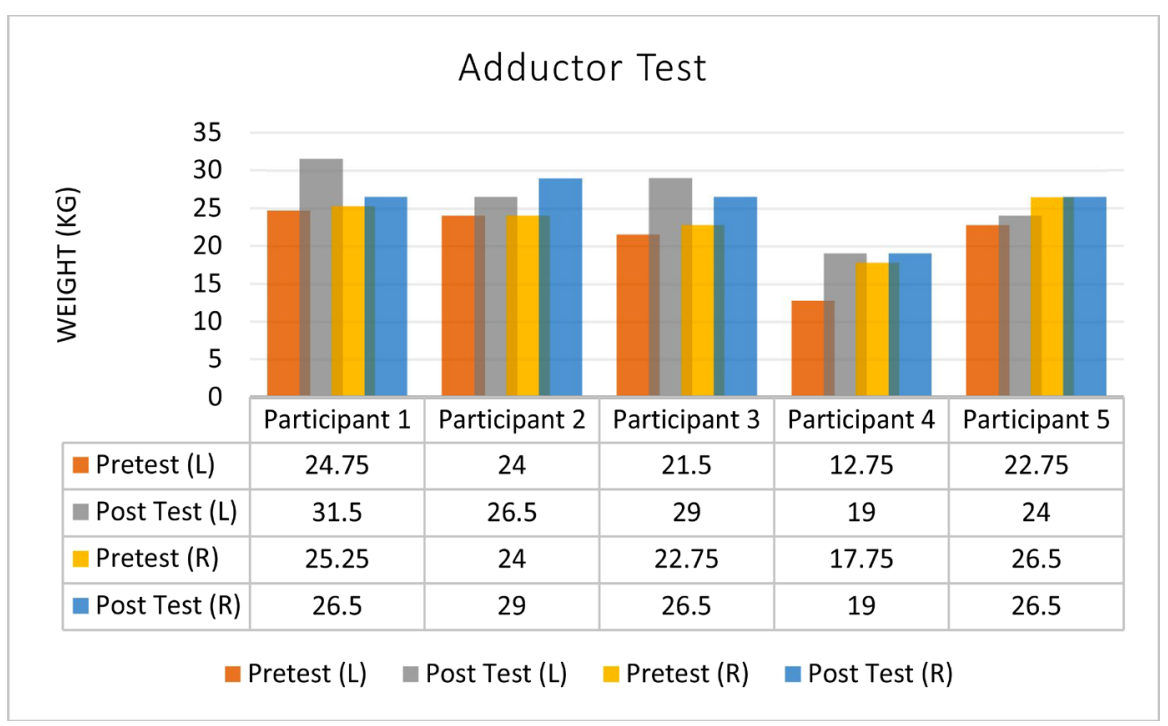

Figure 5. Pre- and post-test results comparison of the single leg adductor test. 


\section{Discussion}

Little is known regarding the exact training protocol for elite squash athletes. The aim of this study was to observe and interpret the results of a 5-week linear periodization training regime on elite squash athletes consisting of 12 SSIPT, 4 Hypertrophy, 3 Speed and Power sessions. The results of our program show that there is a significant increase in post testing performance for all 5 athletes as compared to their pre-test results. For the 1-minute push up, athletes improved at an average of $54.78 \%, 1$ minute sit up test showed an average improvement of $21.5 \%$, half squat test exhibited an improvement of $7.8 \%$, and all round improvements for 20 meter sprint test and adductor test for all 5 participants. Participants show all round improvements in all 5, 10 and $15 \mathrm{~m}$ sprints as well.

This advancement supports the initial hypothesis whereby elite athletes require different variations in their periodisation program to ensure progress is made over a longer period of time (Kraemer, Duncan, \& Volek, 1998). The increase in volume contradicts what is typically seen in a traditional linear periodisation program where generally a taper in volume happens as the program progresses. A study done by (Häkkinen, Pakarinen, Alén, Kauhanen, \& Komi, 1988) has shown that those who have already established a higher pretraining status (meaning to say, high performance athletes) will be limited to the amount of absolute strength gains when compared to the untrained. In contrary from amateurs and medium level athletes who only require low training stimulus to excel, elite athletes enhance their performance through larger amounts of training stimulus which can be tough to obtain from traditional training (Issurin, 2010).

In elite sport, coaches and applied sport scientists are constantly seeking and applying innovative training methods and optimal strategies to have their athletes achieve high performances (Gouttebarge, Moen, Boschman, \& Kantebeen, 2013). To maximize the effectiveness of training, coaches and sport scientists need to evaluate the affects and consequence of training methods on a regular basis through the appropriate training protocols. Doing regular testing and measurement provides coaches and athletes with feedback that can be used to identify the shortcomings of an athlete and adjust training programs to improve those imperfections. Without this feedback, athlete may continue training ineffectively and fail to develop the fitness, skills, tactical awareness, and other capacities needed for competitive success (McAuliffe, 2010).

Reflecting on the data obtained, all athletes managed to show improvement in post test results proving the effectiveness of the program and continues to support previous studies done on elite level adaptations. Essentially coaches can play with training variations of certain fundamental aspects i.e. volume instead of going for more extravagant approaches such as High Intensity Interval Training (HIIT). A study done by (Rynecki, Siracuse, Ippolito, \& Beebe, 2019) shows that there was a $144 \%$ increase in injuries involving both lower and upper body extremities due to the increase in HIIT interest from 2012-2016. Sport Specific In- 
jury Prevention Training (SSIPT), however, was specially implemented in the program and showed no injuries among the athletes while training and post program for SEA Games 2019. Better neuromuscular adaptation was seen with sport specific resistance training as increases in electromyographic EMG amplitude and rate of EMG activation indicates improvements in the pattern of neural drive $(\mathrm{P}<0.05)$ (Judge, Moreau, \& Burke, 2003).

Our training intervention showed an application of Sport Specific Injury Prevention Training (SSPIT) and showed no injuries among the athletes while training and post program for SEA Games 2019. Better neuromuscular adaptation was seen with sport specific resistance training as increases in electromyographic EMG amplitude and rate of EMG activation indicates improvements in the pattern of neural drive $(P<0.05)$ (Judge, Moreau, \& Burke, 2003).

What this study shows is that the methodology of our 5 -week linear periodisation is effective in producing positive results for elite level squash athletes. Due to the limitations of a standard traditional periodisation that raises difficulties for progress in elite athlete's variation needs to be included (Issurin, 2010). This study serves as a guideline to be used as a reference for future studies and training variations to be implemented. Many more in depth studies need to be done with varying training protocols in hopes of getting a baseline of reference for coaches and athletes to refer to. An increase in sample size could prove useful to collect more valid and reliable data with enough statistical power to produce results that reflect a more standardize truth. Comparisons between male and female squash athletes could also serve useful in determining the differences in adaptations between genders

\section{Conclusion}

During the 5-week training program, the athletes underwent a total of 12 SST sessions, 4 hypertrophy sessions and 3 speed and plyometric sessions. All the athletes displayed significant improvement in strength, muscular endurance and speed suggesting that a linear strength and conditioning program can be highly beneficial for squash players. Although the results of the study are positive, further research into various training programs is warranty to further understand the best possible approach for training elite squash players.

\section{Acknowledgements}

Special thanks to Datuk Nicol Ann David and the Singapore Sports Council

\section{Conflicts of Interest}

The authors declare no conflicts of interest regarding the publication of this paper.

\section{References}

Bennie, J., \& Hrysomallis, C. (2005). Resistance Training Considerations for the Sport of 
Squash. Strength and Conditioning Journal, 27, 30-38. https://doi.org/10.1519/00126548-200506000-00005

Docherty, D. (1982). A Comparison of Heart Rate Responses in Racquet Games. British Journal of Sports Medicine, 16, 96-100. https://doi.org/10.1136/bjsm.16.2.96

Fleck, S. (2011). Non-Linear Periodization for General Fitness \& Athletes. Journal of Human Kinetics, 29, 41-45. https://doi.org/10.2478/v10078-011-0057-2

Gouttebarge, V., Moen, M., Boschman, J., \& Kantebeen, M. (2013). Squash-Specific Exercise Test for Elite Squash Players: Development and Validity. European Journal of Sports and Exercise Science, 2, 1-6.

Haff, G. G., \& Nimphius, S. (2012). Training Principles for Power. Strength and Conditioning Journal, 34, 2-12. https://doi.org/10.1519/SSC.0b013e31826db467

Häkkinen, K., Pakarinen, A., Alén, M., Kauhanen, H., \& Komi, P. (1988). Daily Hormonal and Neuromuscular Responses to Intensive Strength Training in 1 Week. International Journal of Sports Medicine, 9, 422-428. https://doi.org/10.1055/s-2007-1025044

Issurin, V. B. (2010). New Horizons for the Methodology and Physiology of Training Periodization. Sports Medicine, 40, 189-206.

https://doi.org/10.2165/11319770-000000000-00000

Judge, L., Moreau, C., \& Burke, J. (2003). Neural Adaptations with Sport-Specific Resistance Training in Highly Skilled Athletes. Journal of Sports Sciences, 21, 419-427.

https://doi.org/10.1080/0264041031000071173

Kraemer, W. J., Duncan, N. D., \& Volek, J. S. (1998). Resistance Training and Elite Athletes: Adaptations and Program Considerations. Journal of Orthopaedic \& Sports Physical Therapy, 28, 110-119. https://doi.org/10.2519/jospt.1998.28.2.110

Rynecki, N. D., Siracuse, B. L., Ippolito, J. A., \& Beebe, K. S. (2019). Injuries Sustained during High Intensity Interval Training: Are Modern Fitness Trends Contributing to Increased Injury Rates? The Journal of Sports Medicine and Physical Fitness, 59, 1206-1212. https://doi.org/10.23736/S0022-4707.19.09407-6

McAuliffe, J. (2010). Beyond the Nick: Long-Term Player Development. https://squash.ca/wp-content/uploads/2020/11/BeyondtheNick_SC_LTPD_Sept8_2010 manual_EN.pdf

van der Kruk, E., van der Helm, F., Veeger, H., \& Schwab, A. (2018). Power in Sports: A Literature Review on the Application, Assumptions, and Terminology of Mechanical Power in Sport Research. Journal of Biomechanics, 79, 1-14. https://doi.org/10.1016/j.jbiomech.2018.08.031

Willoughby, D. (1993). The Effects of Mesocycle-Length Weight Training Programs Involving Periodization and Partially Equated Volumes on Upper and Lower Body Strength. Journal of Strength and Conditioning Research, 7, 2-8. https://doi.org/10.1519/00124278-199302000-00002 


\section{Appendix A}

Table A1. Pre-test: 1RM testing structure used during the study.

\begin{tabular}{ccc}
\hline Number of Set/Reps & $\% 1$ RM & Rest Interval \\
\hline $1 \times 8$ & $40 \%-50 \%$ & 2 minutes \\
$1 \times 5$ & $60 \%-70 \%$ & 2 minutes \\
$1 \times 1$ & $80 \%-90 \%$ & 3 minutes \\
$1 \times 1$ & $95 \%-100 \%$ or New PR & 5 minutes \\
$1 \times 1$ & New PR Attempt & 5 minutes
\end{tabular}

Table A2. Pre-test: strength test results.

\begin{tabular}{cccccc}
\hline \multicolumn{7}{c}{ STRENGTH TEST } \\
\hline Participants & Half Squat & Push up & Sit up & Adductor (L) & Adductor \\
\hline Participant 1 & 160 & 52 & 53 & 24.75 & 25.25 \\
Participant 2 & 110 & 31 & 45 & 24 & 24 \\
Participant 3 & 100 & 55 & 59 & 21.5 & 22.75 \\
Participant 4 & 80 & 13 & 31 & 12.75 & 17.75 \\
Participant 5 & 60 & 10.5 & 51 & 22.75 & 26.5 \\
\hline
\end{tabular}

Table A3. Pre-test: speed test results.

\begin{tabular}{lcccc}
\hline \multicolumn{5}{c}{ 20 m Sprint test } \\
\hline Participants & $5 \mathrm{~m}$ & $10 \mathrm{~m}$ & $15 \mathrm{~m}$ & $20 \mathrm{~m}$ \\
\hline Participant 1 & $1.12 \mathrm{~s}$ & 1.87 & 2.55 & 3.21 \\
Participant 2 & $1.06 \mathrm{~s}$ & 1.84 & 2.54 & 3.21 \\
Participant 3 & $1.03 \mathrm{~s}$ & 1.83 & 2.53 & 3.2 \\
Participant 4 & $1.19 \mathrm{~s}$ & 2.03 & 2.81 & 3.56 \\
Participant 5 & $1.15 \mathrm{~s}$ & 1.97 & 2.72 & 3.43 \\
\hline
\end{tabular}

\section{Appendix B. Hypertrophy Session Breakdown}

Table B1. First hypertrophy session.

\begin{tabular}{cccccc}
\hline \multicolumn{5}{c}{ Hypertrophy Session 1 } \\
\hline EXERCISE & SET & REP & TEMPO & REST & INTENSITY \\
\hline Half Squat & 3 & 8 & 4 sec. Descend & 2 min & $65 \%$ \\
Bench Press & 3 & 8 & 4 sec. Descend & 2 min & $65 \%$ \\
Deadlifts & 3 & 8 & 4 sec. Descend & 2 min & $65 \%$ \\
Overhead Press & 3 & 8 & 4 sec. Descend & 2 min & $65 \%$ \\
Bent Over Rows & 3 & 8 & 4 sec. Descend & 2 min & $65 \%$
\end{tabular}




\section{Continued}

\begin{tabular}{cccccc}
\hline BB Split Squats & 3 & 8 & $4 s e c$. Descend & 2 min & $65 \%$ \\
Banded Hip Ext. & 6 & 8 & $4 s e c$. Descend & 2 min & Light Band \\
Diagonal Sit Ups & 6 & 10 & N/A & $1 \mathrm{~min}$ & BW \\
Banded Dorsi Flex & 6 & 10 & N/A & 1 min & Light Band \\
\hline
\end{tabular}

Table B2. Second hypertrophy session.

\begin{tabular}{cccccc}
\hline \multicolumn{5}{c}{ Hypertrophy Session 2 } \\
\hline EXERCISE & SET & REP & TEMPO & REST & INTENSITY \\
\hline Half Squat & 4 & 8 & 4 sec. Descend & 2 min & $65 \%$ \\
Bench Press & 4 & 8 & 4 sec. Descend & 2 min & $65 \%$ \\
Deadlifts & 4 & 8 & 4 sec. Descend & 2 min & $65 \%$ \\
Overhead Press & 4 & 8 & 4 sec. Descend & 2 min & $65 \%$ \\
Pull Ups & 4 & 8 & 4 sec. Descend & 2 min & $65 \%$ \\
Leg Press & 4 & 8 & 4 sec. Descend & 2 min & $65 \%$ \\
Diagonal Sit Ups & 6 & 10 & N/A & 1 min & BW \\
Banded Dorsi Flex & 6 & 10 & N/A & 1 min & Light Band \\
\hline
\end{tabular}

Table B3. Third hypertrophy session.

\begin{tabular}{cccccc}
\hline \multicolumn{5}{c}{ Hypertrophy Session 3 } \\
\hline EXERCISE & SET & REP & TEMPO & REST & INTENSITY \\
\hline Half Squat & 5 & 8 & 4 sec. Descend & 2 min & $65 \%$ \\
Bench Press & 5 & 8 & 4 sec. Descend & 2 min & $65 \%$ \\
Deadlifts & 5 & 8 & 4 sec. Descend & 2 min & $65 \%$ \\
Overhead Press & 5 & 8 & 4 sec. Descend & 2 min & $65 \%$ \\
Bent Over Rows & 5 & 8 & 4 sec. Descend & 2 min & $65 \%$ \\
BB Split Squats & 5 & 8 & $4 s e c$. Descend & 2 min & $65 \%$ \\
Banded Hip Ext. & 5 & 8 & $4 s e c$. Descend & 2 min & Medium Band \\
Diagonal Sit Ups & 6 & 10 & N/A & 1 min & BW \\
Banded Dorsi Flex & 6 & 10 & N/A & 1 min & Medium Band \\
\hline
\end{tabular}

Table B4. Fourth hypertrophy session.

\begin{tabular}{cccccc}
\hline \multicolumn{7}{c}{ Hypertrophy Session 4 } \\
\hline EXERCISE & SET & REP & TEMPO & REST & INTENSITY \\
\hline Half Squat & 5 & 8 & 4 sec. Descend & 2 min & $75 \%$ \\
Bench Press & 5 & 8 & 4sec. Descend & 2 min & $75 \%$ \\
Deadlifts & 5 & 8 & 4sec. Descend & 2 min & $75 \%$ \\
\hline
\end{tabular}




\section{Continued}

\begin{tabular}{cccccc}
\hline Overhead Press & 5 & 8 & 4 sec. Descend & 2 min & $75 \%$ \\
Pull Ups & 5 & 8 & 4 sec. Descend & 2 min & $75 \%$ \\
Leg Press & 5 & 8 & 4 sec. Descend & 2 min & $75 \%$ \\
Diagonal Sit Ups & 6 & 10 & N/A & 1 min & BW \\
Banded Dorsi Flex & 6 & 10 & N/A & 1 min & Medium Band \\
\hline
\end{tabular}

\section{Power Sessions Breakdown}

Table B5. First power session.

\begin{tabular}{ccccc}
\hline \multicolumn{5}{c}{ POWER SESSION 1 } \\
\hline EXERCISE & SET & REP & REST & INTENSITY \\
\hline Snatch & 4 & 2 & $2 \mathrm{~min}$ & $75 \%$ \\
Snatch Pull & 4 & 2 & $2 \mathrm{~min}$ & $80 \%$ \\
Bench Press & 4 & 2 & $2 \mathrm{~min}$ & $85 \%$ \\
Back Squat & 4 & 2 & $2 \mathrm{~min}$ & $85 \%$ \\
Single-Arm Seated Row & 4 & 3 ea & 2 min & $85 \%$ \\
Single-Leg Barbell Deadlift & 4 & 3 ea & 2 min & $85 \%$ \\
Barbell Calf Raise & 3 & 20 & 1.5 min & $50 \%$ \\
Diagonal Sit Ups & 3 & 20 & 1.5 min & BW \\
\hline
\end{tabular}

Table B6. Second power session.

\begin{tabular}{ccccc}
\hline \multicolumn{5}{c}{ POWER SESSION 2 } \\
EXERCISE & SET & REP & REST & INTENSITY \\
\hline Snatch & 4 & 2 & $2 \mathrm{~min}$ & $80 \%$ \\
Snatch Pull & 4 & 2 & $2 \mathrm{~min}$ & $85 \%$ \\
Bench Press & 4 & 2 & $2 \mathrm{~min}$ & $90 \%$ \\
Back Squat & 4 & 2 & $2 \mathrm{~min}$ & $90 \%$ \\
Single-Arm Seated Row & 4 & 3 ea & 2 min & $90 \%$ \\
Single-Leg Barbell Deadlift & 4 & 3 ea & 2 min & $90 \%$ \\
Barbell Calf Raise & 3 & 20 & 1.5 min & $60 \%$ \\
Diagonal Sit Ups & 3 & 20 & 1.5 min & BW \\
\hline
\end{tabular}


Table B7. Third power session.

\begin{tabular}{ccccc}
\hline \multicolumn{5}{c}{ POWER SESSION 3 } \\
EXERCISE & SET & REP & REST & INTENSITY \\
\hline Snatch & 5 & 2 & 2 min & $80 \%$ \\
Snatch Pull & 5 & 2 & 2 min & $85 \%$ \\
Bench Press & 5 & 2 & 2 min & $90 \%$ \\
Back Squat & 5 & 2 & 2 min & $90 \%$ \\
Single-Arm Seated Row & 5 & 3 ea & 2 min & $90 \%$ \\
Single-Leg Barbell Deadlift & 5 & 3 ea & 2 min & $90 \%$ \\
Barbell Calf Raise & 3 & 20 & 1.5 min & $60 \%$ \\
Diagonal Sit Ups & 3 & 20 & 1.5 min & BW \\
\hline
\end{tabular}

Table B8. Fourth power session.

\begin{tabular}{ccccc}
\hline \multicolumn{5}{c}{ POWER SESSION 4 } \\
\hline EXERCISE & SET & REP & REST & INTENSITY \\
\hline Snatch & 6 & 2 & $2 \mathrm{~min}$ & $80 \%$ \\
Snatch Pull & 6 & 2 & $2 \mathrm{~min}$ & $85 \%$ \\
Bench Press & 6 & 2 & 2 min & $90 \%$ \\
Back Squat & 6 & 2 & 2 min & $90 \%$ \\
Single-Arm Seated Row & 6 & 3 ea & 2 min & $90 \%$ \\
Single-Leg Barbell Deadlift & 6 & 3 ea & 2 min & $90 \%$ \\
Barbell Calf Raise & 3 & 20 & 1.5 min & $60 \%$ \\
Diagonal Sit Ups & 3 & 20 & 1.5 min & BW \\
\hline
\end{tabular}

\section{Appendix C. Retest Results}

Table C1. Strength and endurance retest results following a 5-week program.

\begin{tabular}{cccccc}
\hline \multicolumn{7}{c}{ STRENGTH TEST } \\
\hline Participants & Half Squat & Push up & Sit up & Adductor (L) & Adductor (R) \\
\hline Participant 1 & 166 & 64 & 62 & 31.5 & 26.5 \\
Participant 2 & 122 & 42 & 47 & 26.5 & 29 \\
Participant 3 & 106 & 75 & 77 & 29 & 26.5 \\
Participant 4 & 88 & 29 & 40 & 19 & 19 \\
Participant 5 & 65 & 19.5 & 64 & 24 & 26.5 \\
\hline
\end{tabular}


Table C2. 20-meter sprint retest results following a 5-week program.

\begin{tabular}{lcccc}
\hline \multicolumn{5}{c}{ 20 m Sprint Test } \\
\hline Participants & $\mathbf{5 ~ m}$ & $\mathbf{1 0 ~ m}$ & $\mathbf{1 5 \mathrm { m }}$ & $\mathbf{2 0 ~ \mathrm { m }}$ \\
\hline Participant 1 & $1.03 \mathrm{~s}$ & 1.79 & 2.48 & 3.13 \\
Participant 2 & $1.02 \mathrm{~s}$ & 1.8 & 2.49 & 3.14 \\
Participant 3 & $1.02 \mathrm{~s}$ & 1.78 & 2.48 & 3.15 \\
Participant 4 & $1.09 \mathrm{~s}$ & 2.91 & 2.67 & 3.41 \\
Participant 5 & $1.09 \mathrm{~s}$ & 1.9 & 2.66 & 3.38 \\
\hline
\end{tabular}

\section{Appendix D. Results Comparison}

Table D1. Comparison of Half Squat test results in percentage.

\begin{tabular}{cccc}
\hline \multicolumn{3}{c}{ Half Squat results } \\
\hline Participants & Pretest & Post Test & Improvement in Percentage \\
\hline Participant 1 & 160 & 166 & $3.75 \%$ \\
Participant 2 & 110 & 122 & $10.90 \%$ \\
Participant 3 & 100 & 106 & $6 \%$ \\
Participant 4 & 80 & 88 & $10 \%$ \\
Participant 5 & 60 & 65 & $8.35 \%$ \\
\hline
\end{tabular}

Table D2. Comparison of 1-Minute Push-up Test in percentage.

\begin{tabular}{cccc}
\hline \multicolumn{4}{c}{ 1 Minute Push Up Test } \\
\hline Participants & Pretest & Post Test & Improvement in Percentage \\
\hline Participant 1 & 52 & 64 & $23 \%$ \\
Participant 2 & 31 & 42 & $35.5 \%$ \\
Participant 3 & 55 & 75 & $36.4 \%$ \\
Participant 4 & 13 & 29 & $123 \%$ \\
Participant 5 & 10.5 & 19.5 & $56.0 \%$
\end{tabular}

Table D3. Comparison of 1-Minute Sit-up Test in percentage.

\begin{tabular}{cccc}
\hline \multicolumn{4}{c}{ 1 Minute Sit Up Test } \\
\hline Participants & Pretest & Post Test & Improvement in Percentage \\
\hline Participant 1 & 53 & 62 & $17 \%$ \\
Participant 2 & 45 & 47 & $5 \%$ \\
Participant 3 & 59 & 77 & $30.5 \%$ \\
Participant 4 & 31 & 40 & $30 \%$ \\
Participant 5 & 51 & 64 & $25 \%$
\end{tabular}


Table D4. Comparison of Adductor Strength Test in percentage.

\begin{tabular}{lcccccc}
\hline \multicolumn{7}{c}{ Adductor Test } \\
\hline Participants & Pretest (L) & Pretest (R) & Post Test (L) & $\begin{array}{c}\text { Post Test } \\
\text { (R) }\end{array}$ & L \% & R \% \\
\hline Participant 1 & 24.75 & 25.25 & 31.5 & 26.5 & $27.27 \%$ & $4.95 \%$ \\
Participant 2 & 24 & 24 & 26.5 & 29 & $10.42 \%$ & $20.83 \%$ \\
Participant 3 & 21.5 & 22.75 & 29 & 26.5 & $34.88 \%$ & $16.48 \%$ \\
Participant 4 & 12.75 & 17.75 & 19 & 19 & $49.02 \%$ & $7.04 \%$ \\
Participant 5 & 22.75 & 26.5 & 24 & 26.5 & $5.49 \%$ & $0.00 \%$ \\
\hline
\end{tabular}

Table D5. Comparison of test results of Participant 1.

\begin{tabular}{rccc}
\hline \multicolumn{4}{c}{ 20 Meter Sprint Test-Participant 1 } \\
\\
\hline Pretest & Post Test & Time Increment \\
\hline Meter & 1.12 & 1.03 & 0.09 \\
10 Meter & 1.87 & 1.79 & 0.08 \\
15 Meter & 2.55 & 2.48 & 0.07 \\
20 Meter & 3.21 & 3.13 & 0.08 \\
\hline
\end{tabular}

Table D6. Comparison of test results of Participant 2.

\begin{tabular}{cccc}
\hline \multicolumn{4}{c}{ 20 Meter Sprint Test-Participant 2 } \\
\hline Pretest & Post Test & Time Increment \\
\hline 5 Meter & 1.06 & 1.02 & 0.04 \\
10 Meter & 1.84 & 1.8 & 0.04 \\
15 Meter & 2.54 & 2.49 & 0.05 \\
20 Meter & 3.21 & 3.14 & 0.07 \\
\hline
\end{tabular}

Table D7. Comparison of test results of Participant 3.

\begin{tabular}{cccc}
\hline \multicolumn{4}{c}{ 20 Meter Sprint Test-Participant 3 } \\
\hline Pretest & Post Test & Time Increment \\
\hline 5 Meter & 1.03 & 1.02 & 0.01 \\
10 Meter & 1.83 & 1.78 & 0.05 \\
15 Meter & 2.53 & 2.48 & 0.05 \\
20 Meter & 3.2 & 3.15 & 0.07 \\
\hline
\end{tabular}


Table D8. Comparison of test results of Participant 4.

\begin{tabular}{rccc}
\hline \multicolumn{4}{c}{ 20 Meter Sprint Test-Participant 4} \\
\hline \\
Pretest & Post Test & Time Increment \\
\hline 5 Meter & 1.19 & 1.09 & 0.10 \\
10 Meter & 2.03 & 1.91 & 0.08 \\
15 Meter & 2.81 & 2.67 & 0.14 \\
20 Meter & 3.56 & 3.41 & 0.15 \\
\hline
\end{tabular}

Table D9. Comparison of test results of Participant 5.

\begin{tabular}{lccc}
\hline \multicolumn{4}{c}{ 20 Meter Sprint Test in sec-Participant 5 } \\
\hline Pretest & Post Test & Time Increment \\
\hline 5 Meter & 1.15 & 1.09 & 0.06 \\
10 Meter & 1.97 & 1.9 & 0.07 \\
15 Meter & 2.72 & 2.66 & 0.06 \\
20 Meter & 3.43 & 3.38 & 0.05
\end{tabular}

